\title{
The utility of the AusEd driving simulator in the clinical assessment of driver fatigue
}

\author{
ANUP V. Desai \\ Woolcock Institute of Medical Research, Sydney, New South Wales, Australia \\ and Royal Prince Alfred Hospital, Sydney, New South Wales, Australia \\ BRAD WILSMORE \\ Woolcock Institute of Medical Research, Sydney, New South Wales, Australia \\ DELWYN J. BARTLETT \\ Woolcock Institute of Medical Research, Sydney, New South Wales, Australia \\ and Royal Prince Alfred Hospital, Sydney, New South Wales, Australia \\ Gunnar Unger and Ben Constable \\ Woolcock Institute of Medical Research, Sydney, New South Wales, Australia \\ DAVID JOFFE \\ Woolcock Institute of Medical Research, Sydney, New South Wales, Australia \\ and Royal North Shore Hospital, Sydney, New South Wales, Australia \\ AND \\ Ronald R. GRUNSTEIN \\ Woolcock Institute of Medical Research, Sydney, New South Wales, Australia \\ and Royal Prince Alfred Hospital, Sydney, New South Wales, Australia
}

\begin{abstract}
Several driving simulators have been developed which range in complexity from PC based driving tasks to advanced "real world" simulators. The AusEd"T driving simulator is a PC based task, which was designed to be conducive to and test for driver fatigue. This paper describes the AusEd driving simulator in detail, including the technical requirements, hardware, screen and file outputs, and analysis software. Some aspects of the test are standardized, while others can be modified to suit the experimental situation. The AusEd driving simulator is sensitive to performance decrement from driver fatigue in the laboratory setting, potentially making it useful as a laboratory or office based test for driver fatigue risk management. However, more research is still needed to correlate laboratory based simulator performance with real world driving performance and outcomes.
\end{abstract}

Several driving simulators have been developed to assess driving ability in clinical situations, ranging in complexity from simple PC based driving simulator tasks to advanced "real-world" simulators, where cars mounted on moving bases respond to driver actions (Findley et al., 1989; Haraldsson, Carenfelt, Laurell, \& Tornros, 1990; Juniper, Hack, George, Davies, \& Stradling, 2000). These driving simulators have assessed driving ability in elderly drivers (Gianutsos, 1994), drug affected drivers (Moskowitz \& Robinson, 1990; Smiley, 1986, 1987), and fatigued drivers (Lenne, Triggs, \& Redman, 1998; Philip, Taillard, Quera-Salva, Bioulac, \& Akerstedt, 1999).

Fatigue related road accidents often involve a single vehicle running off the road at high speed; tend to occur on higher speed roadways; and frequently occur at night when there is a circadian increase in sleepiness (Horne \& Reyner, 1995). The AusEd driving simulator, by simulating a monotonous night time drive on a rural road, was developed to create a driving testing environment appropriate for and conducive to driver fatigue. By providing a nonstimulatory or non"alerting" night time driving environment it is hypothesized that the AusEd driving simulator is less likely to mask performance degradation and reduce the tool's sensitivity (Baulk, Reyner LA, \& Horne, 2001; Horne \& Baulk, 2004) in assessing driver sleepiness or fatigue. Most other driving simulator programs used in sleep medicine research to date have used more stimulating protocols to assess driving performance and associated sleepiness (Findley et al., 1989; George, Boudreau, \& Smiley, 1996b; Haraldsson et al., 1990).

A.V.Desai, anupd@med.usyd.edu.au 
For a driving simulator to be utilized as a fatigue risk management device in various settings, such as in sleep laboratories or sleep clinics, or by licensing authorities, it should be simple, inexpensive, and potentially portable. For this reason, $\mathrm{PC}$ based driving simulators are potentially more suitable than the more complex "real-world" simulators, such as those mounted on moving car bases (Haraldsson et al., 1990) as testing tools with clinical applications.

In this article, we describe the AusEd driving simulator and its analysis software, highlight previously published work showing that it is sensitive to performance impairment due to alcohol, sleep deprivation and circadian influences (Banks, Catcheside, Lack, Grunstein, \& McEvoy, 2004, 2005; Desai, Marks, Jankelson, \& Grunstein, 2006; Howard, Gora, Swann, \& Pierce, 2002a, 2002b), and present new data showing simulator performance impairment in participants with obstructive sleep apnea (OSA), which is another major cause of driver sleepiness and impaired driver performance. Finally we speculate on the clinical utility of such a tool in the assessment of fatigued drivers.

\section{METHOD}

\section{Development of the AusEd Driving Simulator}

The AusEd driving simulator began as a joint research project between the Sleep Units of St. Vincent's Sleep Disorders Service and Royal North Shore Hospital in Sydney, Australia, and the Respiratory and Sleep Research Unit at the Royal Infirmary in Edinburgh, Scotland. Development began in 1998 and a statistical package to streamline data analysis was added by the Woolcock Institute of Medical Research in Sydney in 2001. The simulator can be obtained by contacting the Technology Group at The Woolcock Institute of Medical Research, Sydney (e-mail: gdungan@woolcock.org.au).

\section{General Description of the AusEd Computer Program}

A full screen projection of the view from the driver's seat of a car is provided (Figure 1), along with a small speedometer in the upper left hand corner of the screen. The simulator is controlled using accelerator and brake pedals and the steering wheel. The simulated drive takes place on a dual highway at night, with forward vision limited to "low beam" lights. On the road during the drive, slowly moving trucks appear, driving in the same direction as the subject and disappearing when the subject uses the brake. Otherwise there are no other cars on the road. There are no traffic signs or markers apart from regularly placed reflective markers on both edges and in the middle of the road, similar to rural roads in Australia. A continuous, low frequency (approx. $60 \mathrm{~dB}$ ) simulated engine noise is played through the computer speakers for the length of the drive.

\section{Technical Requirements of AusEd Driving Simulator}

\section{Minimum Computer and CPU Requirements}

- Minimum processor requirements are a Pentium II $400 \mathrm{MHz}$ or AMD equivalent.

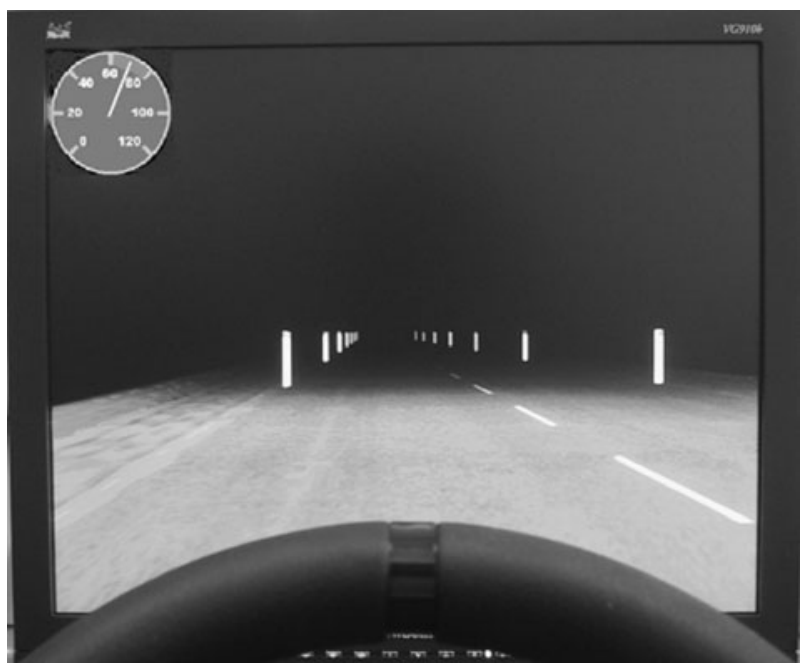

Figure 1. Driver's view of AusEd graphics.

- The minimum RAM requirement is $256 \mathrm{MB}$. This will allow continuous running times of up to $10 \mathrm{~h}$.

- The display should be a minimum of $21 \mathrm{in}$. with a minimum resolution of $640 \times 480$ pixels, running in 256 colors.

- Audio speakers that create an authentic sound (approx. $60 \mathrm{~dB}$ ) in the room where the test is being performed should be selected. There are no special requirements for the audio card and on-board sound cards are sufficient.

\section{Compatibility}

- The program has been validated to run on Windows NT, XP, and 2000 platforms. It does NOT run on Windows 98, $\mathrm{ME}$, or earlier operating systems. It has not been ported to Linux, UNIX, or Macintosh operating systems.

- Any standard steering wheel input interface is usable with the program, using either the game port or via a USB port (Version 1). The basic input device requires a two-pedal system with 1-button activation. There is no activated "force feedback" for this program, and simple steering devices are preferred.

- The video card must be compatible with following video cards for memory mapping purposes:

- Diamond Stealth 64 Video VRAM PCI

- Matrox G200

- Matrox G400.

- For on board video, it will work with Intel Video Chipset.

\section{The Program Module}

\section{Dynamic Outputs}

1. The input device (steering and brake apparatus) determines the start time as well as deriving the analog signal for road position and velocity. The velocity is determined by the degree of accelerator depression and visually simulated by changing the speed of the up-coming road presented to the subject (i.e., the amount of road traveled is a dependent variable of velocity).

2. At low speeds the response to steering is deliberately sluggish and poor redrawing speeds occur to mimic inertia. 


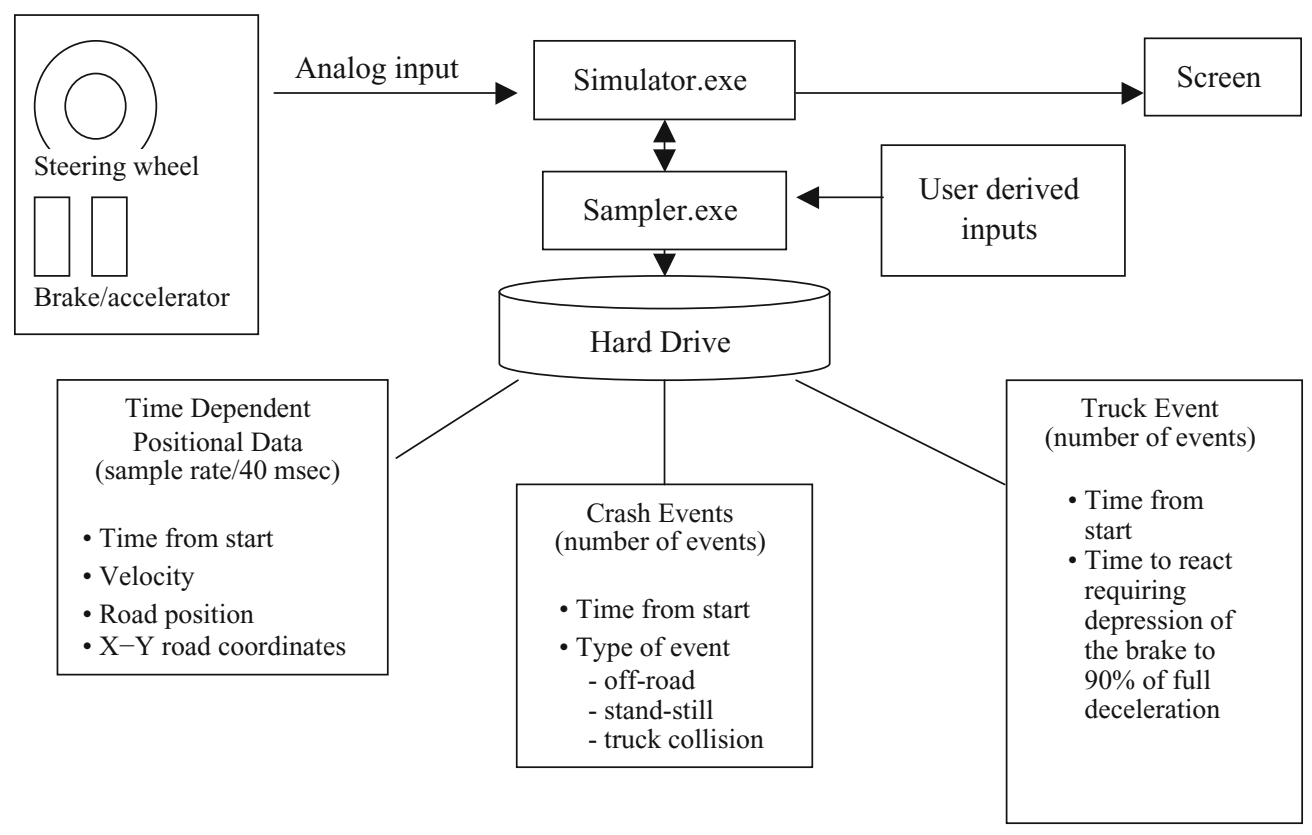

Figure 2. The program module.

\section{Static Inputs}

1. The algorithm for determining road design is derived from the seed number, which can be random (1:4 billion) or predetermined. The seed number will allocate the order of straights and chicanes. The duration of each straight and chicane is derived from the user entered data set in the "Sampler." The drive duration is defined by the user and entered at the time of study set up. The duration must be a minimum of 1 min but can be extended to any time up to $10 \mathrm{~h}$. The time increment can be increased as a metric of a single minute duration.

2. The "Sampler" allows the designation of either right or left lane driving, and the center of the lane is determined by the input variable (i.e., right or left hand drive vehicle). This is then used as the reference (or zero lane) point for the "Simulator" to derive road position and steering deviation. The derived road is drawn from the predetermined measures of lane width $360 \mathrm{~cm}$ and road width $1,440 \mathrm{~cm}$ (including the road shoulders, each of $360 \mathrm{~cm}$ ). The broken center line and the road side reflector poles were derived from real measures of a country road and were mathematically calculated to the simulated road proportions. The sound was recorded from a 240-HP Suzuki motorcycle to a magnetic tape, converted to MPEG format and looped.

3. The number of truck presentations is entered as any given number with a minimum preset of 1 truck. There is no preset maximum for truck presentations. The timing of the truck presentation is a derivative of the seed number. For any given seed number the timing and therefore placement of trucks will be identical across the study period. Altering the seed number and/or the truck number will redefine the truck presentations and their timing.

\section{File Outputs}

1. The output data is recorded and written to disk every $40 \mathrm{msec}$. The basic data set records the time stamp for the sample, the velocity as a $\mathrm{km} / \mathrm{h}$ metric, the $\mathrm{X}-\mathrm{Y}$ coordinate and lane position (in centimeters) as derived from the zero lane position. The $\mathrm{X}-\mathrm{Y}$ coordinate is calculated and recorded as a function of the velocity and the lateral direction (which is a function of road design).

2. The truck presentations are written to the output file. The time taken to $90 \%$ depression of the brake is recorded from the time of presentation of a truck and is written to the output file for each truck. The RT and 1/RT are calculated within the "Sampler" program from this data source and are separately presented following the study termination (see on screen data output).

3. Crash data is recorded by the "Sampler" and written to the data set according to crash type and time. The crash types are defined as follows:

a. Off road. The zero point having been derived from the center of the chosen drive lane will then determine the outer reference points on either side of the road which delimit the drivable boundaries. For example, during a left hand lane drive, the outer boundary for the left hand roadside is $540 \mathrm{~cm}$ and for the right hand roadside is $900 \mathrm{~cm}$. Movement of the reference point outside these boundaries will interrupt the drive and initiate the "crash" warning for the subject. The event time is recorded to the data set.

b. Stand still. Once the simulator has been completely stationary for a period of $10 \mathrm{sec}$, the "crash" warning will be initiated for the subject and the time recorded.

c. Truck collision. The data set records any event whereby the subject collides with the presented truck obstacle. The vehicle needs to maintain a velocity in excess of $60 \mathrm{~km} / \mathrm{h}$ 
for a collision to occur. Deliberate "tail-gating" at speeds below $60 \mathrm{~km} / \mathrm{h}$ will result in a prolonged RT and no further trucks will be presented.

\section{Onscreen Outputs}

1. The onscreen data includes the time and date of the drive, the subject's name, the chosen lane, the duration of the test drive and the number of truck obstacles.

2. The RT and 1/RT is presented for each truck obstacle as well as a mean for the entire test drive.

3 . The mean deviation from the lane center and the mean velocity are calculated and presented.

4. There are 3 derived graphic outputs available.

a. The velocity window visually presents the velocity over the whole drive with a straight line through the center indicating $70 \mathrm{~km} / \mathrm{h}$ and a shaded area around this indicating $60-80 \mathrm{~km} / \mathrm{h}$ (the recommended speed limits).

b. The steering deviation window visually presents the steering deviation from the zero point of the lane over the whole drive. No boundaries are represented.

c. The road map window draws a graphic for the entire road traveled during the test session as derived from the $\mathrm{X}-\mathrm{Y}$ coordinates. This graphic is scalable and can be resized without loss of resolution or distortion.

Each of the above also demonstrate all the crash data according to type and time, and in the case of the road map, the shape of the road when the crash occurred. This forensic analysis is color coded:

$$
\begin{aligned}
& \text { green }=\text { off road crash } \\
& \text { blue }=\text { stand still crash } \\
& \text { red }=\text { truck collision crash }
\end{aligned}
$$

\section{Analysis Module}

A separate module for the output analysis of the AusEd has been created to satisfy research demands. This module provides for more detailed analysis and allows greater flexibility of analysis. The automated on screen output has two major shortcomings. First, the drive is measured in its entirety and cannot be subsegmentally analyzed, which does not allow microanalysis of small data points or brief periods of the study, which may be important. Second, the reaction, which is derived from a $90 \%$ depression of the brake, does not always offer precise data for analysis, particularly if a subject has braked incompletely in response to a truck presentation. The developed analysis module enables subsegmental analysis. It also presents the user with a plot of the velocity versus time after a truck appears and allows for manual marking of the exact time of braking in partitions as small as $40 \mu \mathrm{sec}$. Precise and detailed analysis of each braking event can therefore be provided. To further enhance data analysis, the analysis module also calculates many other outcome variables to supplement the on screen outputs (full list of additional outputs available on request).

The currently used outputs (see Discussion for prior published work) from the analysis module include:

- velocity deviation from the required 60-80 kph speed zone (SPDV). As the speedometer lies outside the line of sight for the road, this represents a divided attention task.

- steering deviation from the center of the left hand lane (STDVC)

- steering deviation from the median lane position during the drive (STDVM). This variable was introduced to take into account differences in interpretation of the "center of the lane" position that are asked to adhere to.

- the mean reaction time (RTMN), median reaction time (RTMD), and the standard deviation of the reaction time (RTSD) for the truck braking episodes

- the number and types of crashes.

\section{AusEd Subject Familiarization and Driving Instructions}

Published studies using other driving simulators have used practice sessions and "acclimatization" periods to reduce learning effects from driving simulators (Shechtman et al., 2006; Turkington, Sircar, Saralays, \& Elliot, 2004). In concordance with this literature and taking into account feedback from participants as to how long it took them to familiarize themselves with the driving task, it is recommended that all subjects first practice the driving simulator during a 5-min test drive. This short practice session should be repeated as many times as necessary until the examiner feels that the subject understands the driving instructions and has become familiar with the driving instrumentation (steering, pedals) and road layout.

Participants are instructed to drive in the center of the left hand lane, to keep their speed between 60 and 80 kilometers per hour (kph), and to brake completely as soon as a truck appears in their line of vision. The truck will then disappear and participants are asked to resume driving as before. As mentioned in The program module description (above), right lane driving is also possible, by altering one of the set up options.

\section{Examining the Effect of Obstructive Sleep Apnea With the AusEd Driving Simulator}

\section{Participant Selection and Protocol}

A prospective study examining AusEd driving simulator performance was performed on 99 participants who underwent polysomnography at Royal Prince Alfred Hospital. Most of the participants had been referred for polysomnography for clinical reasons after a consultation with a Sleep Physician in either a hospital or Medical Centre sleep clinic. A small cohort of control participants was also recruited for the study from advertisements placed at University and hospital campuses.

Participants were eligible if they were 18 to 60 years old, held a current New South Wales driver's license, drove regularly, and were free of significant cardiorespiratory disease, or other medical conditions likely to affect their ability to perform the experimental protocol adequately and safely (e.g., narcolepsy, epilepsy). Participants were ineligible if they were taking any medications or illicit substances that might affect daytime alertness (e.g., antidepressants, benzodiazepines, amphetamines), if they 
were currently being treated or had previous treatment for OSA, or if they were heavy drinkers (regular alcohol consumption greater than $40 \mathrm{~g}$ per day). Commercial drivers were excluded because of their high driving exposure.

The protocol was approved by the Central Sydney Area Health Service Ethics Committee (RPA zone) and the University of Sydney Ethics Committee and informed consent was obtained from each participant.

Participants were permitted to smoke and to continue their usual caffeine intake in the days prior to attending the sleep laboratory. However, after arrival in the sleep laboratory caffeine products and cigarette smoking were not allowed for the testing duration. On the evening of admission to the sleep laboratory, participants completed a short questionnaire (physical and demographic information, information regarding caffeine intake, driving and lifestyle, and the Epworth Sleepiness Scale (Johns, 1991), were familiarized with and practiced the AusEd driving task and were given at least a 30-min break before conducting a full $30 \mathrm{~min}$ AusEd trial at $7 \mathrm{pm}$. Participants were then set up for overnight polysomnography as previously described in our laboratory (Desai, Marks, \& Grunstein, 2003). Lights out was at 10:15 pm, and the participants slept, while being monitored, until 6 am. Participants then showered, had breakfast (no caffeine), and commenced their final AusEd testing at $7 \mathrm{am}$.

For this experiment, the driving simulator task was set to $30 \mathrm{~min}$, with 10 trucks appearing during this time, and $5 / 7$ of the road was straight while $2 / 7$ of the road consisted of chicanes. The layout of the road and time of presentation of trucks was identical for each drive.

\section{Statistical Analysis}

In all AusEd analyses, the first six minutes of the drive was not analyzed to further reduce any potential for learning effects (Hack et al., 2000). The data was analyzed using a mixed model ANOVA, which was consistent with other studies in this area. Caffeine and age were included as continuous covariates in the ANOVA (i.e., ANCOVA). Caffeine was calculated as follows: number cups of coffee $+0.5 *$ (number of cups tea $)+0.5 *$ (number of cans cola). Groupings were performed post hoc, based on the respiratory disturbance index (RDI) and Epworth sleepiness scores (ESS). The control group did not have OSA (RDI < 5) and were not suffering daytime sleepiness $(\mathrm{ESS} \leq 10)$. Obstructive sleep apnea syndrome (OSAS)
Table 1

Participant Characteristics

\begin{tabular}{llccc}
\hline & & $N$ & $M$ & $S D$ \\
\hline Age (years) & Controls & 28 & 40 & 13 \\
& Intermediate & 53 & 46 & 13 \\
& OSAS & 13 & 42 & 12 \\
BMI $\left(\mathrm{kg} / \mathrm{m}^{2}\right)$ & Controls & 28 & 27.5 & 5.0 \\
& Intermediate & 53 & 30.7 & 6.2 \\
& OSAS & 13 & 35.9 & 6.4 \\
RDI (events/h) & Controls & 28 & 2 & 2 \\
& Intermediate & 53 & 19 & 20 \\
ESS & OSAS & 13 & 58 & 29 \\
& Controls & 28 & 4 & 3 \\
& Intermediate & 53 & 8 & 6 \\
& OSAS & 13 & 16 & 4 \\
\hline
\end{tabular}

Note-BMI, body mass index; RDI, respiratory disturbance index; ESS, Epworth sleepiness score; OSAS, obstructive sleep apnea syndrome.

group had moderate to severe OSA (RDI $\geq 15)$ and were symptomatic $($ ESS $>10)$. Participants falling between these two groups were considered intermediate. These criteria were based on evidenced based guidelines (American Academy of Sleep Medicine Task Force, 1999), clinical applicability (George, 2004), and previous studies in the area where daytime sleepiness has also been used as part of the grouping criteria of OSA patients (Findley, Suratt, \& Dinges, 1999; Haraldsson et al., 1990; Juniper et al., 2000). Data were tested for normal distribution. The number of crashes was not normally distributed, subsequently crash frequency was log transformed (after the addition of 0.5 to all values of zero to be transformed). Post hoc was analysis was performed using least significant difference. Alpha was set at .05 for all analyses.

All data analyses described above were undertaken using the SPSS statistical package 10.0.05 (SPSS Inc., Chicago).

\section{Examining the Reproducibility of the AusEd Driving Simulator}

Data from a previously published study which examined AusEd driving performance in control participants on two separate occasions under identical conditions (Desai et al., 2006) were further examined to determine reproducibility of the AusEd. In this study, men were studied exclusively; all testing was at the same time (7 pm); participants had similar driving exposure as described above; participants were requested to sleep at least 8 hours per

Table 2

Two-Way ANOVAs [3 (group) $\times 2$ (Time)] for All AusEd Performance Outcomes

\begin{tabular}{|c|c|c|c|c|c|c|c|}
\hline \multirow[b]{2}{*}{ Dependent } & \multicolumn{2}{|c|}{$\begin{array}{c}\text { Time } \\
(d f=1)\end{array}$} & \multicolumn{2}{|c|}{$\begin{array}{l}\text { Interaction } \\
(d f=2) \\
\end{array}$} & \multicolumn{2}{|c|}{$\begin{array}{c}\text { Group } \\
(d f=2)\end{array}$} & \multirow[b]{2}{*}{ Significance } \\
\hline & $p$ & $F$ & $p$ & $F$ & $p$ & $F$ & \\
\hline Steering deviation (STDVM) & .886 & 0.02 & .011 & 4.76 & .001 & 7.42 & $\mathrm{O}>\mathrm{I}=\mathrm{C}$ \\
\hline Speed deviation (SPDV) & .233 & 1.44 & .843 & 0.17 & .446 & 0.82 & $\mathrm{O}=\mathrm{I}=\mathrm{C}$ \\
\hline Reaction time (RTMN) & .653 & 0.20 & .232 & 1.49 & .004 & 6.01 & $\mathrm{O}>\mathrm{I}=\mathrm{C}$ \\
\hline Reaction time (RTSD) & .687 & 0.16 & .394 & 0.94 & .003 & 6.22 & $\mathrm{O}=\mathrm{I}>\mathrm{C}$ \\
\hline Crashes* & .388 & 0.75 & .074 & 2.69 & .023 & 3.97 & $\mathrm{O}>\mathrm{I}=\mathrm{C}$ \\
\hline
\end{tabular}


night for the 4 nights preceding each driving test; and actigraphy was used to objectively measure these prior sleep hours. Reproducibility results are presented as mean and standard deviations to represent the bias and precision, and the significance of any differences were assessed by paired $t$ tests.

\section{RESULTS}

\section{Examining the Effect of Obstructive Sleep Apnea With the AusEd Driving Simulator}

The participant characteristics are presented in Table 1, with each characteristic presented according to group. Ninety-four participants had complete data suitable for analysis. As expected from the definitions of the groups, RDI and ESS values increased progressively from controls to intermediate to OSAS groups. There was 1 woman in the study. Her values were not statistically different from the men.

Table 2 and Figure 3 present the results from the ANOVA analyses, controlled for age and caffeine intake. The OSAS participants were worse than controls on 4 out of 5 AusEd performance outcomes, and worse than the intermediate group on 3 out of 5 outcomes. Intermediate participants were worse than controls on one variable.

\section{Steering Deviation}

OSAS participants had significantly more lane variability $(152.4 \mathrm{~cm}$, confidence interval [CI] 123.0 to 181.9$)$ compared with both controls $(p<.001 ; 102.9 \mathrm{~cm}, \mathrm{CI}$ 82.8 to 123.0$)$ and intermediate $(p=.007 ; 88.6 \mathrm{~cm}, \mathrm{CI}$ 74.0 to 103.3$)$. Lane position variability did not change over time $(p=.886)$. However, there was a significant interaction between group and time ( $p=.001)$, such that the OSAS group were much worse in the evening testing relative to the morning testing, compared with both intermediate and control participants.

\section{Speed Variability}

Unlike lane position, there was no difference between groups $(p=.446)$, no effect of time $(p=.233)$ and no group by time interaction $(p=.843)$.

\section{Reaction Time-Mean}

The OSAS group $(1,551.5 \mathrm{msec}$, CI 1,370.1 to 1,732.9) had significantly longer reaction times compared with controls ( $p=.001 ; 1,171.3 \mathrm{msec}$, CI $1,047.5$ to $1,295.1)$ and compared with the intermediate group ( $p=.008$; $1271.8 \mathrm{msec}$, CI 1181.7 to 1362.0 ). There was no effect of time ( $p=.653)$, or interaction $(p=.232)$.

\section{Reaction Time - Standard Deviation}

The OSAS group (536.2 msec, CI 378.0 to 694.4) had significantly greater reaction time variability compared with controls ( $p=.001 ; 211.0 \mathrm{msec}$, CI 103.0 to 318.9) but not compared with the intermediate group ( $p=.076$; $376.1 \mathrm{msec}$, CI 297.4 to 454.6$)$. There was no effect of time $(p=.687)$, or interaction $(p=.394)$.

\section{Crash Frequency}

The OSAS group (0.56, CI 0.29 to 0.83 ) had significantly more crashes compared with the controls ( $p=$ $.006 ; 0.07, \mathrm{CI}-0.12$ to 0.25$)$ and with the intermediate group ( $p=.049 ; 0.22$, CI 0.09 to 0.36$)$. There was no effect of time $(p=.388)$, or interaction $(p=.074)$.

\section{Reproducibility}

Participant characteristics for this analysis are presented in Table 3.

Table 4 shows that there were no significant differences between tests performed on consecutive days.

\section{DISCUSSION}

In this article we have described the development and details of the AusEd driving simulator, a new PC based driving task, with potential clinical utility. The AusEd driving task simulates a monotonous night time drive on a rural Australian road, an environment that is conducive to driver fatigue or sleepiness. Although some aspects of the driving task are necessarily standardized (e.g., night driving environment, minimal curbside or road distractions/ obstacles, hardware and analysis), other aspects can be readily modified to suit different experimental conditions (e.g., left or right hand driving, the total time of the driving simulation, the number of truck obstacles and the nature of the road [the number of straight vs. chicane sections]).

Two previously published studies have demonstrated that the AusEd driving simulator is sensitive to performance decrement from fatigue promoting factors (Banks et al., 2004; Desai et al., 2006). In the study by Banks et al., partial sleep deprivation and low levels of blood alcohol (mean serum concentration $0.035 \pm 0.015 \mathrm{~g} / \mathrm{dl}$ ) impaired driving simulator performance compared with partial sleep deprivation alone. Time-on-task effects were also seen for some of the driving simulator outcomes. A more recent paper from this same study has shown that performance on the AusEd driving simulator correlates with the Maintenance of Wakefulness Test sleep latency, another commonly used measure of sleepiness (Banks et al., 2005). In the study by Desai et al., the AusEd driving simulator was sensitive to circadian influences on performance, as well as to the effects of acute sleep deprivation. Other studies, published in abstract form, show that poor AusEd driving simulator performance correlates with slow eye closure, EEG and PVT measures of sleepi-

Table 3

Participant Characteristics for the Control Group in AusEd Reproducibility Testing $(N=13)$

\begin{tabular}{ccc}
\multicolumn{3}{c}{ Reproducibility Testing $(\boldsymbol{N}=\mathbf{1 3})$} \\
\hline Subject Characteristic & $M$ & \multicolumn{1}{c}{$S D$} \\
\hline Age (years) & 37.0 & 14.6 \\
BMI (kg/m $\left.{ }^{2}\right)$ & 25.5 & 5.4 \\
RDI (events/h) & 1.7 & 1.4 \\
ESS & 7.2 & 4.6 \\
\hline
\end{tabular}

Note-BMI, body mass index; RDI, respiratory disturbance index; ESS, Epworth sleepiness score. 

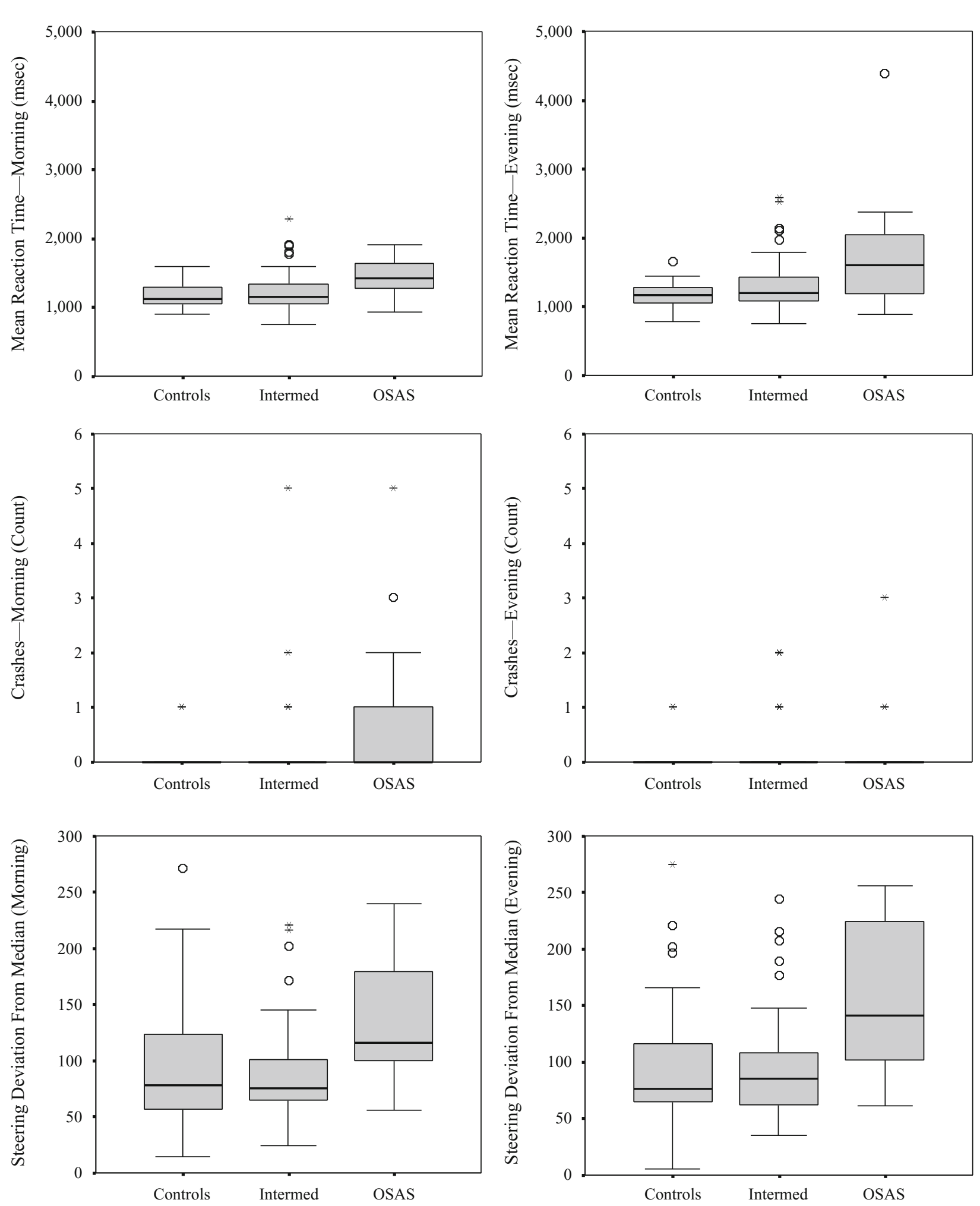

Figure 3. Box plot of AusEd outcomes by group. The box length is the interquartile range. Circles are outliers i.e., cases with values between 1.5 and 3 box lengths from the upper or lower edge of the box. Asterisks are extreme cases i.e., cases with values more than 3 box lengths from the upper or lower edge of the box. 
Table 4

AusEd Performance Results for the Control Group

\begin{tabular}{lccccccccc}
\hline & \multicolumn{2}{c}{ First Test } & & \multicolumn{2}{c}{ Second Test } & & \multicolumn{2}{c}{ Difference } & \\
\cline { 2 - 3 } \multicolumn{1}{c}{ Variable } & $M$ & $S D$ & & $M$ & $S D$ & & $M$ & $S D$ & $p$ \\
\hline STDVM $(\mathrm{cm})$ & 42.8 & 9.1 & & 40.8 & 12.8 & & 1.9 & 10.3 & .513 \\
SPDV $(\mathrm{km} / \mathrm{h})$ & 1.3 & 1.0 & & 1.4 & 1.3 & & -0.1 & 0.7 & .651 \\
RTMN $(\mathrm{msec})$ & $1,160.9$ & 136.4 & & $1,158.3$ & 197.0 & & 2.5 & 116.8 & .939 \\
RTSD $(\mathrm{msec})$ & 194.7 & 81.5 & & 218.6 & 191.3 & & -23.9 & 183.6 & .647 \\
Number of crashes & 0.08 & 0.28 & & 0.00 & 0.00 & & 0.08 & 0.28 & .337 \\
\hline
\end{tabular}

Note - Results were obtained on two consecutive days, at the same time of day $(N=13)$.

See Table 2 for an explanation of variables.

ness (Howard et al., 2002a, 2002b), and that the AusEd driving simulator is sensitive to performance improvement after Modafinil in sleep deprived mild OSA patients (Newcombe et al., 2001). This article adds to the existing published literature by showing that the AusEd driving simulator is sensitive to performance decrement due to obstructive sleep apnea, a common medical cause of driver sleepiness or fatigue.

Therefore, with the addition of this paper, studies now show that the AusEd driving task is sensitive to performance decrement due to several common driver fatigue promoting factors - sleep deprivation (Desai et al., 2006), alcohol (Banks et al., 2004, 2005), circadian influences (Desai et al., 2006), time-on-task effects (Banks et al., 2004), and sleep disorders (present study). Data presented in this article also show that the test results are reproducible. In order to further demonstrate the clinical utility of this driving task, it will be important to show that this tool can detect an improvement in performance when sleep apnea patients are treated. Such changes have been shown for other driving simulators (George, Boudreau, \& Smiley, 1997; Munoz, Mayoralas, Barbe, Pericas, \& Agusti, 2000; Ortho et al., 2002). This needs to be the subject of further research.

The experimental data presented in this paper has some limitations. First, it is possible that there is selection bias due to the nonrandomized enrolment of OSA and control patients. In addition the study did not significantly control for driving exposure (though commercial drivers were excluded), an important factor affecting driving performance (George, 2003). However, the study did control for other factors known to affect simulator performance (age, caffeine intake) and only men were studied (gender has been shown to affect driving performance).

Table 2 and Figure 3 show that the AusEd driving simulator discriminates the OSAS group from the 'intermediate' and control groups in terms of driving performance. However, there is a large overlap between individuals of the groups, with some OSAS participants performing as well as controls and others clearly performing worse. This has also been noted previously in similar simulator studies (George, 2000; George, Boudreau, \& Smiley, 1996a) and may reflect the fact that not all drivers with OSAS have on road collisions or are unsafe drivers (George, 2003). To date, there is no single test or group of tests that can be used to reliably predict poor on road driving performance/ accident risk in fatigued drivers. Driving simulators are potential tools that could in the future prove useful for this.
However, before they can be confidently used, there needs to be data showing that those drivers who perform poorly on simulators are the ones who have on road collisions. To date, there is a limited amount of data to support this with a few studies showing that driving simulators can assess driving impairment due to alcohol and drug consumption and in elderly drivers as reliably as on-road studies (Lee, Cameron, \& Lee, 2003; Moskowitz H et al., 1990; Smiley, 1986, 1987). More research is needed to investigate this final link between laboratory based simulator testing and real world driving in fatigued drivers.

In conclusion, we have described a new performance assessment tool, the AusEd driving simulator, which replicates an environment conducive to driver fatigue and has been shown to be sensitive to performance impairment from driver fatigue in a laboratory setting. As a PC based task, it may have widespread applicability as a fatigue management device to detect the impaired sleepy driver. However, before it can be widely used for this purpose, more research is needed, including linking poor driving simulator performance to poor on road driving performance in fatigued drivers.

\section{AUTHOR NOTE}

B.C., D.J., and R.R.G. are part owners of the copyright of the AusEd software. Correspondence concerning this article should be addressed to A. V. Desai, E11, Royal Prince Alfred Hospital, Missenden Rd., Camperdown, Sydney, 2050 Australia (e-mail: anupd@med.usyd.edu.au).

\section{REFERENCES}

American Academy of Sleep Medicine Task Force (1999). Sleeprelated breathing disorders in adults: Recommendations for syndrome definition and measurement techniques in clinical research. Sleep, 22, 667-689.

Banks, S., Catcheside, P., Lack, L., Grunstein, R. R., \& McEvoy, R. D. (2004). Low levels of alcohol impair driving simulator performance and reduce perception of crash risk in partially sleep deprived subjects. Sleep, 27, 1063-1067.

Banks, S., Catcheside, P., Lack, L., Grunstein, R. R., \& McEvoy, R. D. (2005). The maintenance of wakefulness test and driving simulator performance. Sleep, 28, 1381-1385.

Baulk, S., Reyner, L. A., \& Horne, J. A. (2001). Driver sleepinessEvaluation of reaction time measurement as a secondary task. Sleep, 24, 695-698.

Desai, A. V., Marks, G., \& Grunstein, R. (2003). Does sleep deprivation worsen mild obstructive sleep apnea? Sleep, 26, 1038-1041.

Desai, A. V., Marks, G., Jankelson, D., \& Grunstein, R. R. (2006). Do sleep deprivation and time of day interact with mild obstructive sleep apnoea to worsen performance and neurobehavioural function. Journal of Clinical Sleep Medicine, 2, 63-70.

Findley, L., Fabrizio, M., Knight, H., Norcross, B., Laforte, A., 
\& SURATT, P. M. (1989). Driving simulator performance in patients with sleep apnea. American Review of Respiratory Disease, 140 529-530.

Findley, L., Suratt, P. M., \& Dinges, D. F. (1999). Time-on-task decrements in "steer clear" performance of patients with sleep apnea and narcolepsy. Sleep, 22, 804-809.

George, C. F. (2000). Vigilance impairment: Assessment by driving simulators. Sleep, 23, S115-S118.

George, C. F. (2003). Driving simulators in clinical practice. Sleep Medicine Reviews, 7, 311-320.

George, C. F. (2004). Driving and automobile crashes in patients with obstructive sleep apnoea/hypopnoea syndrome. Thorax, 59, 804-807.

George, C. F., Boudreau, A. C., \& Smiley, A. (1996a). Comparison of simulated driving performance in narcolepsy and sleep apnea patients. Sleep, 19, 711-717.

George, C. F., Boudreau, A. C., \& Smiley, A. (1996b). Simulated driving performance in patients with obstructive sleep apnea. American Journal of Respiratory \& Critical Care Medicine, 154, 175-181.

George, C. F., Boudreau, A., \& Smiley, A. (1997). Effects of nasal CPAP on simulated driving performance in patients with obstructive sleep apnoea. Thorax, 52, 648-653.

Gianutsos, R. (1994). Driving advisement with the elemental driving simulator (EDS): When less suffices. Behavior, Research Methods, Instruments, \& Computers, 26, 183-186.

Hack, M. A., Davies, R. J., Mullins, R., Choi, S., RamdassinghDow, S., Jenkinson, C., ET AL. (2000). Randomised prospective parallel trial of therapeutic versus subtherapeutic nasal continuous positive airway pressure on simulated steering performance in patients with obstructive sleep apnoea. Thorax, 55, 224-231.

Haraldsson, P. O., Carenfelt, C., Laurell, H., \& Tornros, J. (1990). Driving vigilance simulator test. Acta Otolaryngology (Stockholm), 110, 136-140.

HoRne, J. A., \& REYNER, L. A. (1995). Sleep related vehicle accidents British Medical Journal, 310, 565-567.

HoRne, J., \& BAULK, S. (2004). Awareness of sleepiness when driving. Psychophysiology, 41, 161-165.

Howard, M., Gora, J., Swann, P., \& Pierce, R. (2002a). Alpha and theta activity and slow eye closure are related to driving performance in professional drivers. Sleep, 25(Abstract Supplement), A148.

Howard, M., Gora, J., Swann, P., \& Pierce, R. (2002b). Evidence for poor perception of sleepiness in professional drivers. Sleep, $\mathbf{2 5}$ (Abstract Supplement), A146.

JoHNS, M. W. (1991). A new method for measuring daytime sleepiness: The Epworth Sleepiness Scale. Sleep, 14, 540-545.

Juniper, M., Hack, M. A., George, C. F., Davies, R. J., \& Stradling,
J. R. (2000). Steering simulation performance in patients with obstructive sleep apnoea and matched control subjects. European Respiratory Journal, 15, 590-595.

Lee, H., Cameron, D., \& Lee, A. (2003). Assessing the driving performance of older adult drivers: On-road versus simulated driving. Accident Analysis \& Prevention, 35, 797-803.

Lenne, M. G., Triggs, T. J., \& Redman, J. R. (1998). Interactive effects of sleep deprivation, time of day, and driving experience on a driving task. Sleep, 21, 38-44.

Moskowitz, H. \& Robinson, C. D. (1990). The effects of low doses of alcohol on driving related skills: A review of the evidence. (Rep. No. DOT HS-807-280). Springfield, VA: National Technical Information Service.

Munoz, A., Mayoralas, L., Barbe, F., Pericas, J., \& Agusti, A. (2000). Long term effects of CPAP on daytime functioning in patients with sleep apnoea syndrome. European Respiratory Journal, 15, 676-681.

Newcombe, J., Desai, A., Joffe, D., Engleman, H., Seale, J., \& Grunstein, R. (2001). Modafinil improves alertness and driving simulator performance in sleep-deprived mild obstructive sleep apnoea (OSA) subjects. Sleep, 24(Abstract Supplement), A260.

Ortho, M., Leidag, M., Kotterba, S., Widdig, W., De Zeeuw, J., WALTHER, J., ET AL. (2002). Estimation of accident risk in obstructive sleep apnoea syndrome (OSAS) by driving simulation. Pneumologie, 56.

Philip, P., Taillard, J., Quera-Salva, M. A., Bioulac, B., \& AKERSTEDT, T. (1999). Simple reaction time, duration of driving and sleep deprivation in young versus old automobile drivers. Journal of Sleep Research, 8, 9-14.

Shechtman, O., Classen, S., Stephens, B., Davis, E., Bendixen, R., BELCHIOR, P., ET AL. (2006). The impact of intersection design on simulated driving performance of young and senior adults. Topics in Geriatric Rehabilitation, 22, 27-35.

Smiley, A. (1986). Marijuana: On-road and driving simulator studies. Alcohol, Drugs \& Driving, 1, 3-4.

Smiley, A. (1987). Effects of tranquillizers and antidepressants on psychomotor performance. Journal of Clinical Psychiatry, 48, 22-29.

Turkington, P., Sircar, M., Saralays, D., \& Elliot, M. (2004). Time course of changes in driving simulator performance with and without treatment in patients with sleep apnoea hypopnoea syndrome. Thorax, 59, 56-59.

(Manuscript received January 29, 2006 revision accepted for publication September 4, 2006.) 